\title{
Antimalarial activity of novel imidazolidinedione derivatives
}

\author{
Anna Jaromin ${ }^{1,}{ }^{*}$, Anna Czopek ${ }^{2}$, Agnieszka Zagórska 2 , Silvia Parapini ${ }^{3}$, Nicoletta Basilico ${ }^{4}$, Jerzy Gubernator \\ ${ }^{1}$ Department of Lipids and Liposomes, Faculty of Biotechnology, University of Wroclaw, Wroclaw, Poland \\ ${ }^{2}$ Department of Medicinal Chemistry, Jagiellonian University Medical College, Cracow, Poland \\ ${ }^{3}$ Dipartimento di Scienze Biomediche per la Salute, Università degli Studi di Milano, Milan, Italy \\ ${ }^{4}$ Dipartimento di Scienze Biomediche, Chirurgiche e Odontoiatriche, Università degli Studi di Milano, Milan, Italy
}

\section{Background}

Malaria is caused by protozoan parasites of the species Plasmodium. Malaria parasites have developed resistance to chemotherapeutic agents such as chloroquine (CO), mefloquine, and sulfadoxine/pyrimethamine. Therefore, an urgent need exists to develop new classes of antimalarial drugs that operate by nove mechanisms of action. Chlorproguanil is highly active against primary exoerythrocytic forms of $P$. falciparum and $P$. vivax. Moreover, a cyclic dicarboxamide derivative of chlorproguanil, compound WR182393 (2-guanidinoimidazolidinedione derivative) was found to completely eliminate malaria parasites from the body [1]. Several peptide and amino acid derivatives of primaquine and other 8-aminoquinoline antimalarials have been synthesized with the aim of reducing the metabolic oxidative deamination pathway, as well as to reduce toxicity of the parent drug. Moreover, imidazolidin-4-ones prepared from amino acid derivatives of primaquine exhibit potent gametocytocidal activity against $P$. berghei [2]. Systematic structure-activity relationship studies undertaken on a hit compound TDR32750 (4-oxo-4,5-dihydro-1H-pyrrole-3carboxylate derivative), with the aim of improving antiparasitic activity, revealed that replacement of the 4-oxo-4,5dihydro-1 $\mathrm{H}$-pyrrole core on the imidazolidin-2,4-dione gave a similar level of activity against $P$. falciparum [3].

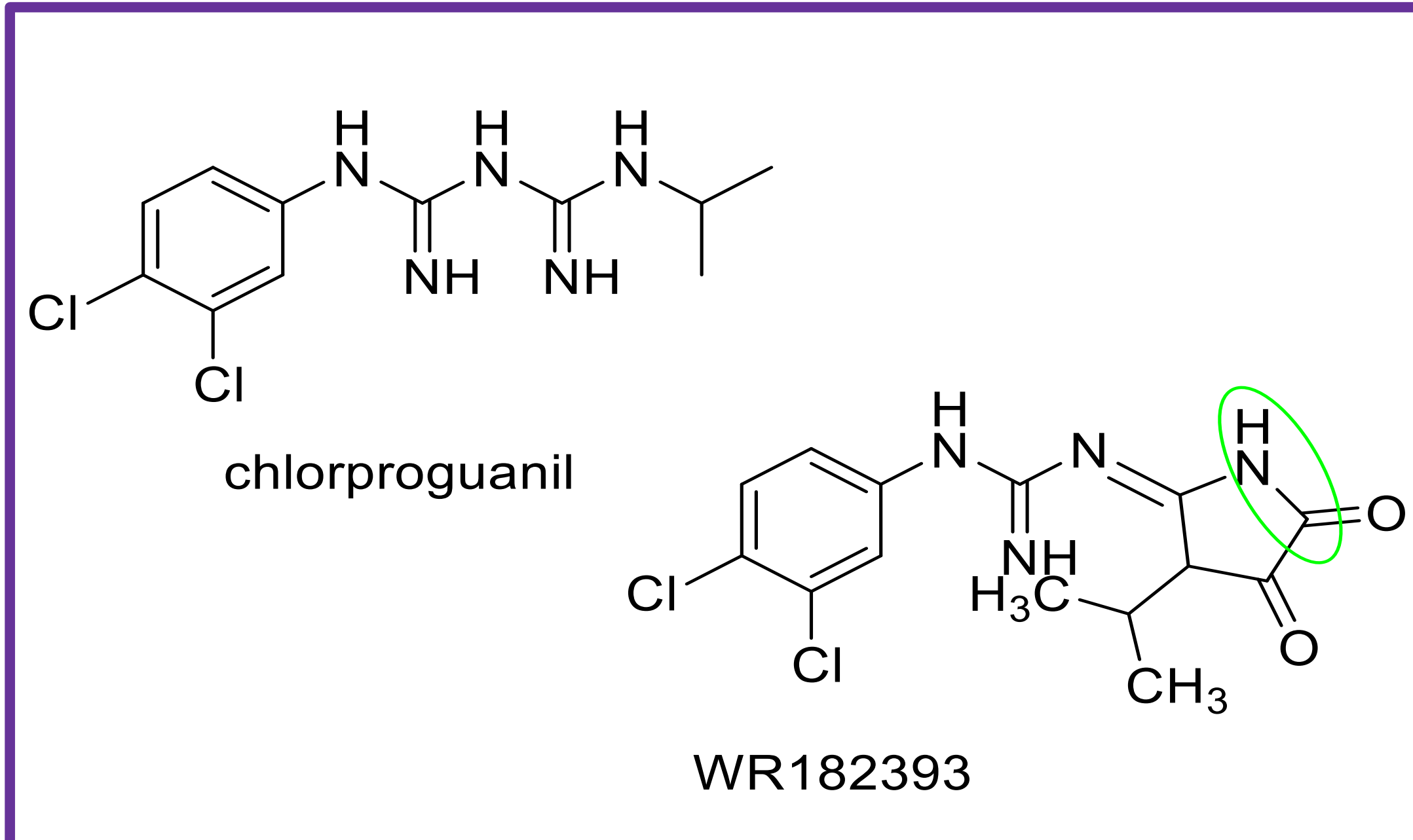

Objective

The aim of the presented study was the evaluation of antimalarial activity of novel imidazolidinedione derivatives.

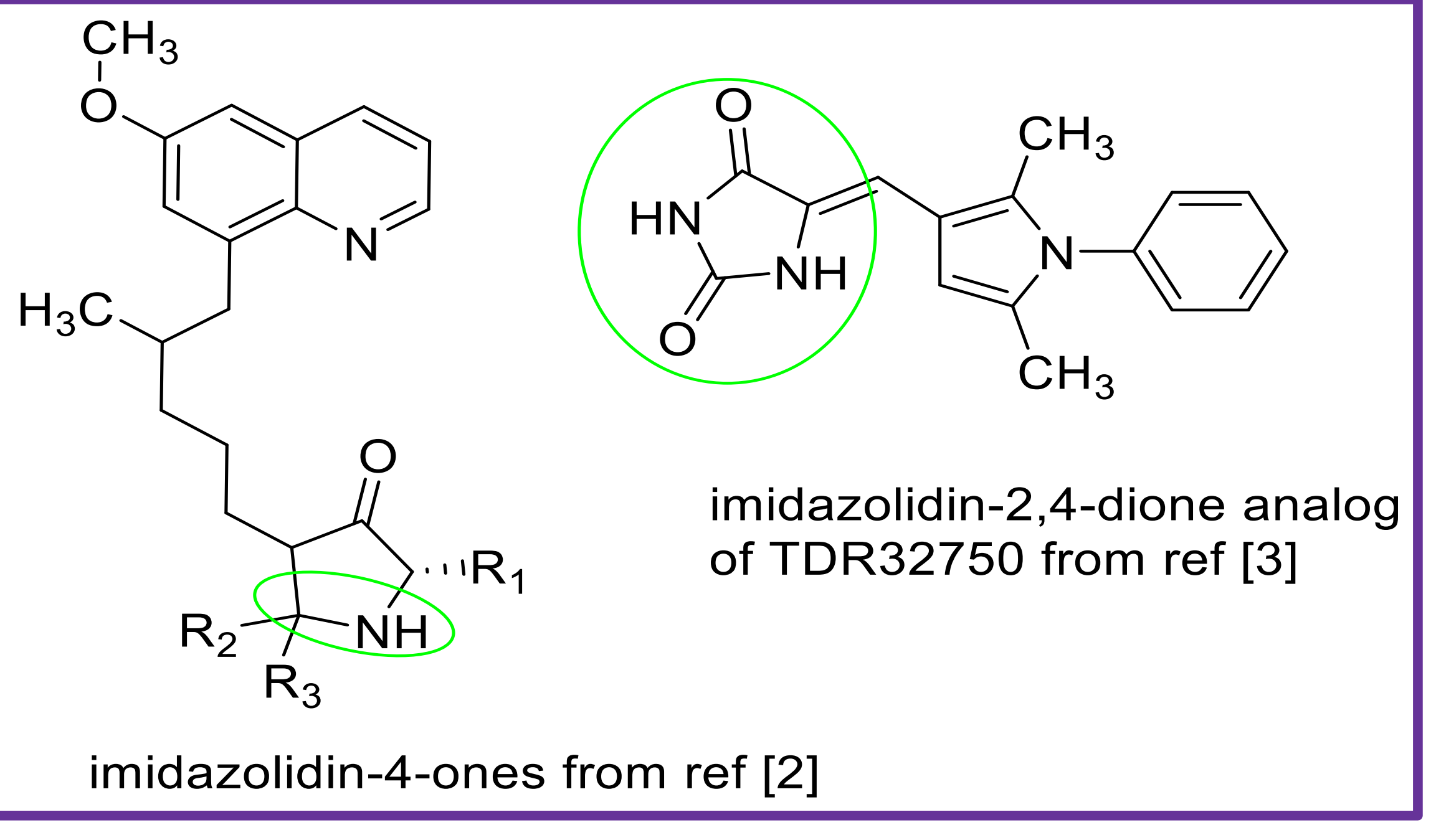

Chemistry

The starting imidazolidinedione rings were prepared from appropriate ketones, 3,4-dihydro-naphthalen-1(2H)-one and 2,3-dihydro- $1 H$-inden-1-one, using the Bucherer-Bergs reaction, with modifications described by Goodson et al. The intermediate products of imidazolidinedione were obtained through an alkylation reaction with 1,5-dibromopentane. In the final step, the intermediate products were coupled with an arylpiperazine moiety to derive the desired compounds (Acz 100-101 and Acz 108-109).

P. falciparum cultures were established according to Trager and Jensen, with slight modifications [4]. Parasite growth was determined spectrophotometrically $\left(\mathrm{OD}_{650}\right)$ by measuring the activity of parasite lactate dehydrogenase (pLDH), according to a modified version of the method of Makler [5]. Antimalarial activity was expressed as $50 \%$ inhibitory concentrations $\left(\mathrm{IC}_{50}\right)$. Results

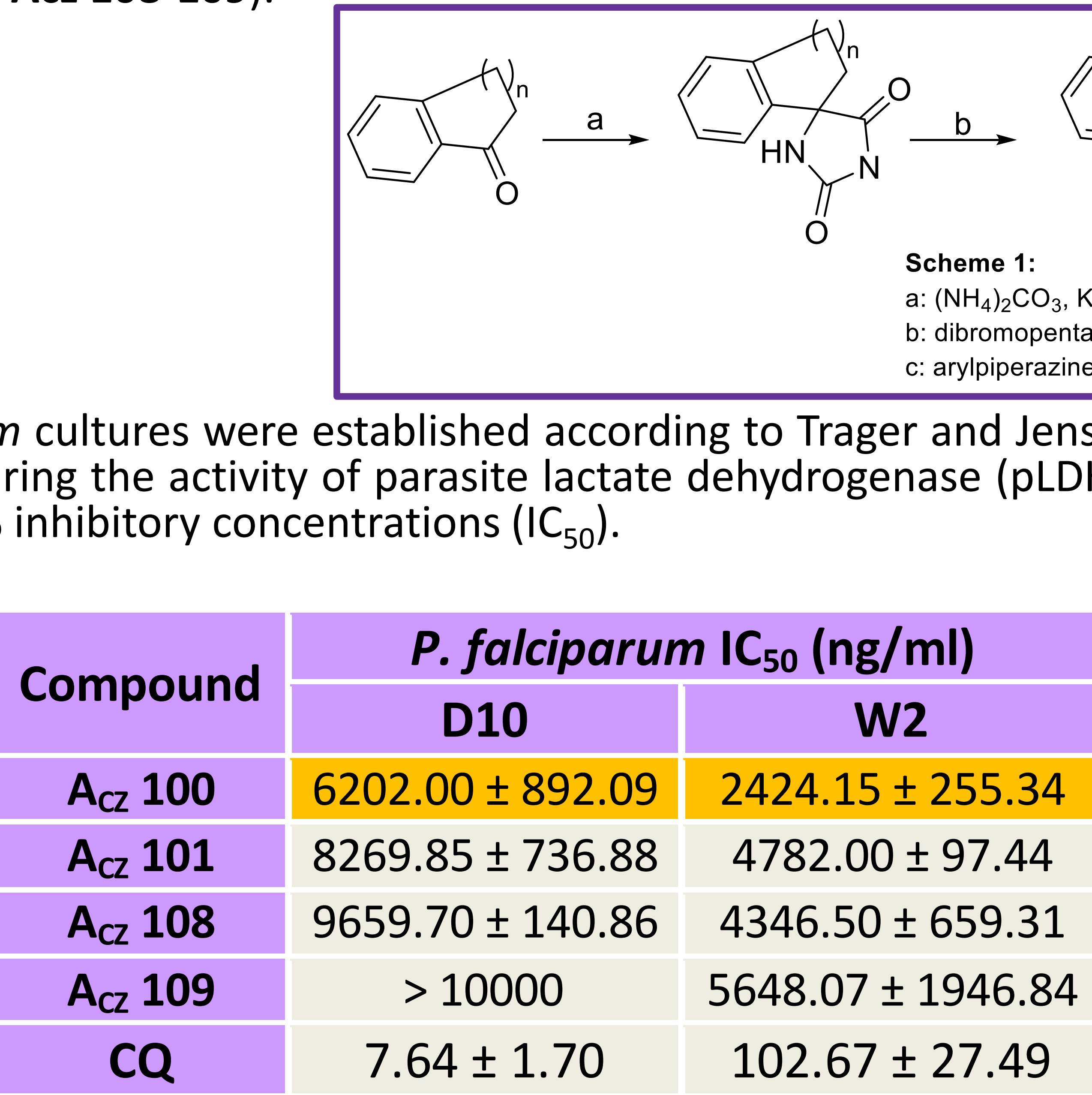

In vitro antimalarial activity of Acz compounds against the D10 (CQ-sensitive) and W2 (CQ-resistant) strains of $P$. falciparum. Conclusion

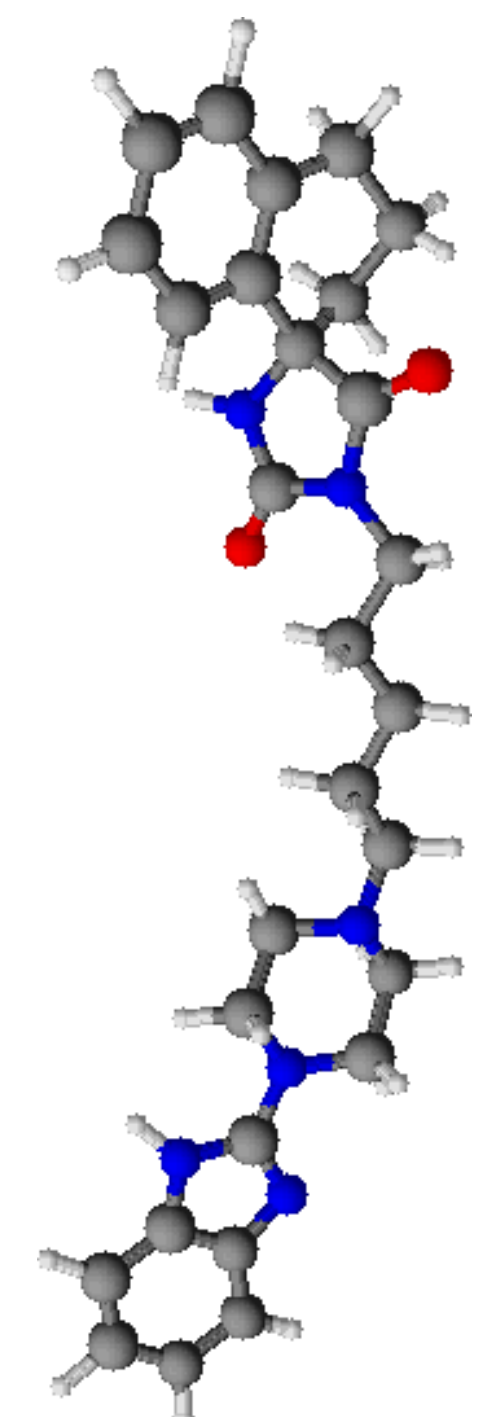

3D-structure of the most active compound, Acz 100

The synthetized derivatives, possessing enhanced antimalarial activity against the CQ-resistant strain of $P$. falciparum, are promising antimalarial drug candidates. The results also indicate the need for development of appropriate lipid delivery systems due to the highly hydrophobic nature of these active compounds.

1.Guan J, Zhang Q, Montip G, Karle JM, Ditusa CA, Milhous WK, Skillman DR, Lin AJ. Structure identification and prophylactic antimalarial efficacy of 2-guanidinoimidazolidinedione derivatives. Bioorg. Med. Chem. 2005,13, 699-704. 2.Araújo MJ, Bom J, Capela R, Casimiro C, Chambel P, Gomes P, lley J, Lopes F, Morais J, Moreira R, de Oliveira E, do Rosário V, Vale N. Imidazolidin-4-one derivatives of primaquine as novel transmissionblocking antimalarials. J. Med. Chem. 2005, 48, 888-892. 3.Mital A, Murugesan D, Kaiser M, Yeates C, Gilbert IH. Discovery and optimisation studies of antimalarial phenotypic hits. Eur. J. Med. Chem. 2015, 103, 530-538. 4.Trager, W., Jensen, J.B. Human malaria parasites in continuous culture Science. 1976, 193, 673-675.5.Makler, M.T, Hinrichs, D.J. Measurement of the lactate dehydrogenase activity of Plasmodium falciparum as an assessment of parasitemia. Am. J. Trop. Med. Hyg. 1993 48, 205-210.

The project is co-financed by the Polish National Agency for Academic Exchange, Italian Ministry of Foreign Affairs and International Cooperation "Executive Programme for Scientific and Technological Cooperation between the Italian Republic and the Republic of Poland and supported by JUMC grant no N42/DBS/000178. 